\title{
Identification of Nanoscale Dissipation Processes by Dynamic Atomic Force Microscopy
}

\author{
R. Garcia, ${ }^{1, *}$ C. J. Gómez, ${ }^{1}$ N. F. Martinez, ${ }^{1}$ S. Patil, ${ }^{1}$ C. Dietz, ${ }^{2}$ and R. Magerle ${ }^{2}$ \\ ${ }^{1}$ Instituto de Microelectrónica de Madrid, CSIC, Isaac Newton 8, 28760 Tres Cantos, Madrid, Spain \\ ${ }^{2}$ Chemische Physik, Technische Universität Chemnitz, D-09107 Chemnitz, Germany
}

(Received 11 April 2006; published 7 July 2006)

\begin{abstract}
Identification of energy-dissipation processes at the nanoscale is demonstrated by using amplitudemodulation atomic force microscopy. The variation of the energy dissipated on a surface by a vibrating tip as a function of its oscillation amplitude has a shape that singles out the dissipative process occurring at the surface. The method is illustrated by calculating the energy-dissipation curves for surface energy hysteresis, long-range interfacial interactions and viscoelasticity. The method remains valid with independency of the amount of dissipated energy per cycle, from 0.1 to $50 \mathrm{eV}$. The agreement obtained between theory and experiments performed on silicon and polystyrene validates the method.
\end{abstract}

DOI: 10.1103/PhysRevLett.97.016103

The emergence of moving micro and nanomechanical devices where the separation between relevant surfaces is in the nanometer range and, in particular, of scanning probe methods, anticipated the importance of energydissipation processes at the nanoscale [1-3]. The amount of energy dissipated between two interacting nanostructures can be accurately measured by atomic force microscopy (AFM) and surface force apparatus methods. However, in most of the cases, those measurements do not clarify the nature of the dissipation process. On the other hand, it is emerging that direct or indirect effects of dissipative processes could be a powerful ally to achieve atomic, molecular,and nanoscale contrast with dynamic atomic force microscopy (AFM) for a wide variety of materials such as semiconductors, oxides, polymers,or biomolecules [3-12].

Dissipation at the nanoscale may involve surface elastic and plastic deformation and/or matter removal. Unwanted surface modification implies invasiveness, loss of contras$\mathrm{t}$, and resolution which penalizes the performance of a high resolution microscope. However, dissipation at the nanoscale could also be accomplished in a wearless regime which would be compatible with high resolution imaging. This is, in particular, the case for amplitude-modulation AFM (AM-AFM), where a powerful and widely used method to detect compositional variations, phase contrast imaging, relies on the detection of changes in the energy dissipated in a local region of the sample surface [13-19]. However, the development of an AFM-based dissipation pectroscopy to provide quantitative analysis of material properties has been hampered by the incomplete knowledge of the dissipative process at the nanoscale.

In this Letter we propose a method to identify the mechanism of energy dissipation at the nanoscale. The method requires the determination of the energy dissipated on the sample surface as a function of the oscillation amplitude while the tip approaches the surface. The representation of the dissipated energy and, in particular, its derivative with respect to the amplitude, dynamicdissipation curves hereafter, characterizes the dissipation
PACS numbers: 68.37.Ps, 07.79.Lh, 62.25.+g, 87.64.Dz

process. Three different nonconservative processes are studied: surface energy hysteresis, viscoelasticity, and long-range dissipative interfacial interactions. The quantitative and qualitative agreement obtained between calculations and experiments performed on silicon and polystyrene samples supports the validity of the identification method proposed here.

Dissipation in atomic force microscopy can be described at the level of atoms and molecules [20,21] or at the nanoscale $[4,22,23]$. In the first case, the emphasis is placed on the specific atomic processes that allow the transformation of mechanical energy from the tip in atomic and molecular motions in the sample. At the nanoscale, which is the focus of this Letter, the emphasis is placed on the quantitative relationship between dissipation and macroscopic quantities such as surface adhesion energy, elastic modulus, stiffness, plasticity index, or viscoelasticity.

We have used a point-mass model to calculate the properties and interactions of a vibrating tip in the proximity of a surface. Point-mass models have demonstrated great quantitative accuracy to describe amplitude-modulation AFM experiments whenever the contribution of higher cantilever eigenmodes can be neglected [24,25]. The simulations were obtained by solving numerically the equation of motion with a fourth order Runge-Kutta algorithm [12].

Let us consider the steady state of a vibrating tip that interacts with the sample surface through conservative (short and long-range forces) and nonconservative interactions. Then, the energy per cycle supplied by the external force $\left(E_{\text {ext }}\right)$ must be converted into either hydrodynamic damping in the medium $\left(E_{\mathrm{med}}\right)$ or energy dissipated in the sample $\left(E_{\mathrm{dis}}\right)$. Furthermore, in many situations of interest, the tip motion can be described by a sinusoidal function $z=z_{0}+A \cos (\omega t-\phi)$ [25]. Those assumptions allow us to express the energy dissipated (per cycle) on the sample surface in terms of experimental quantities,

$$
E_{\mathrm{dis}}=E_{\mathrm{ext}}-E_{\mathrm{med}}=\frac{\pi k A}{Q}\left(A_{0} \sin \phi-\frac{A \omega}{\omega_{0}}\right),
$$

where $E_{\text {med }}$ is modeled by a linear viscous damping law 
$\left(F_{\text {med }}=-b \dot{z}\right) ; Q$ is the quality factor of the cantilever; $A_{0}$ is the amplitude very far from the surface, and $\Phi$ is the phase shift between the external excitation and the tip response. The above equation is equivalent to the one deduced in Ref. [13].

The sample deformation and its associated stress is calculated by the Derjaguin-Muller-Toporov (DMT) model [26], i.e.,

$$
F_{\mathrm{DMT}}=Y^{*} R^{1 / 2} \delta^{3 / 2}-4 \pi R \gamma,
$$

then the energy dissipated by surface energy hysteresis processes can be calculated by,

$$
E_{\mathrm{dis}}=\oint F_{\mathrm{DMT}} d z=4 \pi R \delta\left(\gamma_{r}-\gamma_{a}\right)
$$

Where $\delta$ is the deformation (indentation) and $F_{\mathrm{DMT}}$ is the DMT forces in approach and retraction half periods; $\gamma_{r}$ and $\gamma_{a}$ are, respectively, the approach and retraction surface energies and $Y^{*}$ is the effective elastic modulus of the interface and $R$ is the tip radius.

Long-range dissipative interfacial forces, i.e., interactions that do not imply the mechanical contact between probe and surface (noncontact) are calculated by using a time-dependent power law interaction where the strength of the force $\alpha$ depends on whether the probe approaches $\left(\alpha_{a}\right)$ or retracts away $\left(\alpha_{r}\right)$ from the surface,

$$
F_{i}=-\frac{\alpha(t)}{d^{2}}
$$

then the energy dissipated per cycle is calculated by,

$$
\begin{aligned}
E_{\mathrm{dis}} & =\oint \frac{\alpha}{z^{2}} d z=\int_{d_{1}}^{d_{2}} \frac{\alpha_{a}}{z^{2}} d z-\int_{d_{1}}^{d_{2}} \frac{\alpha_{r}}{z^{2}} d z \\
& =\Delta \alpha\left(\frac{1}{d_{1}}-\frac{1}{d_{2}}\right),
\end{aligned}
$$

where $d_{1}$ and $d_{2}$ are, respectively, the closest and the farthest tip-surface separation during a cycle. The viscoelastic behavior is calculated by using the Voigt model and by assuming that contact area and sample deformation are calculated by the Hertz contact mechanics. The above assumptions give a time-dependent viscous force as,

$$
F_{v}=\eta \sqrt{R \delta} \frac{d \delta}{d t}
$$

where $\eta$ is the viscosity.

Single beam silicon cantilevers (Nanosensors, Germany) with spring constants $k$ of 2 and $35 \mathrm{~N} / \mathrm{m}$ and $Q$ of 150 and 800 , respectively, were used to perform the experiments. The force constant was determined by characterizing the hydrodynamic response of the cantilever [27]. The cantilever was oscillated at its free resonance frequency ( 59 or $285 \mathrm{kHz}$ ) with a free amplitude $A_{0}$ in the 3-60 $\mathrm{nm}$ range. Nominal tip radius were $10 \mathrm{~nm}$ unless otherwise stated. All the experiments were performed at room temperature and at a relative humidity of about $30 \%$. Silicon samples were used after a treatment in
$\mathrm{H}_{2} \mathrm{O}_{2}: \mathrm{NH}_{4} \mathrm{OH}: \mathrm{H}_{2} \mathrm{O}$ (1:1:2) which passivates the $\mathrm{Si}(100)$ surface with a $0.6 \mathrm{~nm}$ film of silicon dioxide. For this surface we have taken $Y=150 \mathrm{GPa}, \alpha_{a}=6.7 \times$ $10^{-29} \mathrm{~J} \cdot \mathrm{m}$ and $\alpha_{r}=100 \mathrm{~mJ} / \mathrm{m}^{2} ; \alpha_{r}=2.5 \alpha_{a}$ and $\gamma_{r}=$ $170 \mathrm{~mJ} / \mathrm{m}^{2}$ [28]. Polystyrene (PS) and polybutadiene (PB) were obtained from Polymer Source Inc. (Canada) with weight-averaged molecular weights of $M_{w}=34.3 \mathrm{~kg} / \mathrm{mol}$ and $M_{w}=365 \mathrm{~kg} / \mathrm{mol}$, and a polydispersity of $M_{w} / M_{n}=$ 1.04 and $M_{w} / M_{n}=1.12$, respectively. A blend of 20:80 (PS:PB) has been spun cast from a toluene solution on a polished silicon wafer. The blend separates into isolated domains of PS surrounded by a PB matrix. PS is characterized by $\eta=800 \mathrm{~Pa} \cdot \mathrm{s}$ and $Y=1 \mathrm{GPa}$ [29].

The amplitude and phase shift dependencies of the oscillation on tip-sample separation were obtained by approaching the tip towards the sample from a distance with negligible tip-sample interaction. Both the change of the oscillation amplitude and the phase shift were recorded as the tip-sample distance was modified. The curves were taken at $2 \mathrm{~Hz}$. Then, Eq. (1) was used to turn data into dissipation values.

Figure 1 shows the calculated dynamic-dissipation curves for the processes examined here. For each curve, the dissipated energy has been normalized with respect to its maximum value $\left[E^{*}=E_{\mathrm{dis}} / E_{\mathrm{dis}}(\max )\right]$. The derivative has been calculated with an algorithm that takes four adjacent points. We also plot the data versus the amplitude ratio $A / A_{0}$.

A dissipation mechanism associated with long-range interfacial interactions described by Eq. (4) is characterized by a near symmetric energy-dissipation curve with a maximum $\left(A_{0}=10 \mathrm{~nm}, k=2 \mathrm{~N} / \mathrm{m}\right.$, squares $)$. The representation of the closest tip-surface distance while the tip approaches the surface shows a minimum with respect to the amplitude ratio. That minimum $d_{\min }$ controls the position of the maximum because the energy is integrated over an interval where the force has practically vanished at the upper end of the oscillation. Mathematically, $2 A \sim d_{2} \gg$ $d_{1}$ and $1 / d_{1} \gg 1 / d_{2}$. Consequently, the energy is con-
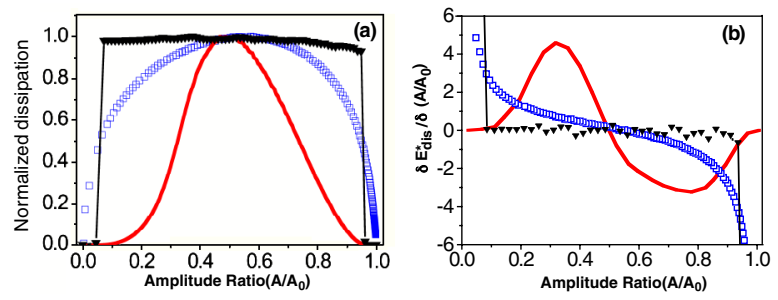

FIG. 1 (color online). (a) Dynamic-dissipation curves for different nonconservative interactions (simulations). Energydissipation values are calculated per oscillation cycle. Triangles are for surface energy hysteresis; squares for a (noncontact) interfacial interaction, and the solid line is for viscoelasticity. Each curve has been normalized with respect to its respective maximum, $E_{\mathrm{dis}}(\max )=9.2,1.4$, and $50 \mathrm{eV}$, respectively. (b) Derivative with respect to the amplitude ratio of the curves shown in (a). 
trolled by the value of $d_{1}$, and it will show a maximum with respect to $A / A_{0}$ for the smallest $d_{1}$ [Eq. (3)]. The derivative shows two sections, one concave up for small $A / A_{0}$ ratios and another concave down for high amplitude ratios [squares in Fig. 1(b)]. Both sections meet in an inflection point situated around $A / A_{0} \sim 0.5$.

Surface energy hysteresis generates energy-dissipation curves characterized by a plateau covering almost $95 \%$ of $A / A_{0}$ values except for those very close to the edges where the dissipation changes dramatically from the plateau value to zero $\left(A_{0}=40 \mathrm{~nm}, k=2 \mathrm{~N} / \mathrm{m}\right.$, triangles $)$. Actually, a zoom on the plateau region reveals the presence of a small maximum (not shown). According to Eq. (2), the shape of the dynamic-dissipation curve for surface energy hysteresis processes is dominated by the sample deformation. This is also supported by the simulations; however, the indentation does not increase linearly by decreasing the amplitude ratio, i.e., with the reduction of the average tip-surface separation. Actually, the simulations for materials with $Y$ in the 10 to $200 \mathrm{GPa}$ range show a small maximum when the indentation is plotted with respect to $A / A_{0}$. The above maximum coincides with the position of the maximum barely visible in the energy-dissipation curves [Fig. 1(a)]. Typical indentation values for $\mathrm{Si}$ and $A_{0}$ in the $20-40 \mathrm{~nm}$ range are about $\sim 0.1 \mathrm{~nm}$. Dissipation increases slowly with $A_{0}$, but more sharply for compliant materials because lowering the elastic modulus increases the deformation. For materials with $Y=10 \mathrm{GPa}$ we obtain indentation values of about $0.5 \mathrm{~nm}$ while recording dissipation curves $\left(A_{0}=40 \mathrm{~nm}\right)$. The derivative is flat for most of $A / A_{0}$ range. The flat section is limited by two sharp changes. Those changes reflect the transition from the attractive regime (conservative here) to the repulsive regime (nonconservative) and vice versa [30].

Dynamic-dissipation curves for viscoelastic materials are rather complex because the viscous force depends on both the indentation and how the indentation changes with time $\left(A_{0}=10 \mathrm{~nm}, \quad k=40 \mathrm{~N} / \mathrm{m}, \quad Y=0.5 \mathrm{GPa}, \quad \eta=\right.$ $500 \mathrm{~Pa} \cdot \mathrm{s}$, solid line). The simultaneous dependence on the indentation and its rate gives them a characteristic feature, the presence of inflection points. Dissipation is very small at both ends of the $A / A_{0}$ range because there the sample deformation is small and consequently the viscous force [Eq. (6)]. The maximum happens for the $A / A_{0}$ that maximizes the product between the indentation and its rate. The derivative shows two extreme points which reflect the different evolution of the indentation and its rate on the amplitude ratio.

The identification method proposed here has been tested experimentally. In Fig. 2 we provide a comparison between theory and experiments performed on silicon and on a PS region of a blend of PS/PB polymer [31]. For all the cases studied, the experimental dynamic-dissipation curves reproduce the behavior predicted by the theory. Energydissipation curves for long-range interactions and viscoelasticity may look similar [Figs. 2(a) and 2(c)], but the derivative unambiguously singles out each dissipative pro-

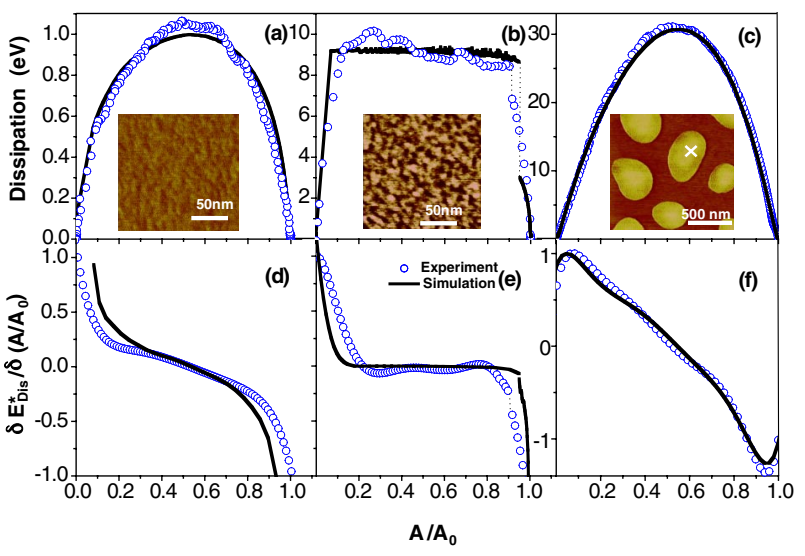

FIG. 2 (color online). Measured and simulated dynamicdissipation curves. (a) On a $\mathrm{Si}$ surface when there is not mechanical contact between tip and surface; $A_{0}=6.6 \mathrm{~nm}, k=$ $2 \mathrm{~N} / \mathrm{m}$. (b) On silicon when there are surface energy hysteresis and long-range interfacial interactions; $A_{0}=32.5 \mathrm{~nm}, k=$ $2 \mathrm{~N} / \mathrm{m}$. (c) On a PS region (cross in the inset) of PS/PB blend, $A_{0}=15 \mathrm{~nm}$. (d),(e),(f) The derivatives of the normalized energy-dissipation curves shown in (a),(b),(c), respectively. The insets show the energy-dissipation images taken on $\mathrm{Si}$, Figs. 2(a) (attractive) and 2(b) (repulsive), and on a PS region [Fig. 2(c)]. The steplike discontinuities observed in Fig. 2(b) mark the transition between attractive and repulsive interaction regimes.

cess [Figs. 2(d)-2(f)]. The simulations presented in Figs. 2(b) and 2(e), consider both long-range dissipative interactions and surface energy hysteresis because the experimental data shows both dissipation in the attractive and repulsive regimes of the AM-AFM. The steplike discontinuities observed in Fig. 2(b) mark the transition between attractive and repulsive regimes (discontinuous line). Those transitions give rise to sharp peaks in the derivative; however, for clarity purposes the peaks are not plotted [30].

In most of the data, the experiments and the theory differ by less than $1 \%$. The larger differences are always below $10 \%$. The differences could be attributed to the influence of in-plane dissipative forces. Measurements performed on organic monolayers have shown that in-plane friction could account for about $8 \%$ of the total dissipated energy in AM-AFM [32].

The input parameters $\left(R, Y, \eta, A_{0}, Q, k\right.$, and $\left.\omega_{0}\right)$ for the model have been taken from the experimental values. It is worth noting that the quantitative agreement happens in rather different ranges of experimental amplitudes $A_{0}=$ 6.6, 15, and $32.5 \mathrm{~nm}$ for noncontact dissipation [Fig. 2(a)], viscoelasticity [Fig. 2(c)], and surface energy hysteresis [Fig. 2(b)], respectively. For high resolution AM-AFM parameters, i.e., $A_{0}$ in the range between 5 and $30 \mathrm{~nm}$ and $R$ between 2 and $10 \mathrm{~nm}$ dissipation values for longrange interfacial interactions, surface energy hysteresis, and viscoelestacity are in the $0.1-2 \mathrm{eV}, 5-20 \mathrm{eV}$, and $20-50 \mathrm{eV}$ ranges, respectively. The quantitative agreement 
obtained between simulations and experiments can be considered a key step to develop a method for analysis of material properties based on dissipation measurements.

It could be argued that energy-dissipation measurements performed by loading and unloading the tip (static measurements) should give the same results that dynamicdissipation experiments whenever the load-unload rate coincides with the frequency of the oscillation. However, a major difference lies in how the mechanical energy stored in the cantilever is converted into potential energy in the sample (indentation), hydrodynamic damping, and dissipation. The deformation in surface energy hysteresis, the minimum distance in long-range interfacial interactions and the deformation and its rate in viscoelasticity are parameters that depend on tip-surface conservative and nonconservative interaction forces, and on the tip's dynamics. It is this interdependence, which does not exist in static measurements, that supports the ability of dynamic methods to identify the dissipation process at the nanoscale.

The work needed to separate two surfaces in the presence of surface adhesion hysteresis is always greater than the originally gained by bringing the surfaces together. The atomistic and molecular mechanisms responsible for this behavior depend on both material properties and environment. They may involve force-induced atomic or molecular reorientations, interdigitation, exchange of atoms and molecules or charged-induced dissipation effects. We have demonstrated that the derivative of the dissipated energy as a function of the amplitude can discriminate between long and short-range surface adhesion hysteresis processes. Those nonconservative processes together with viscoelasticity are among the most common dissipation mechanisms at the nanoscale which render a general validity to the identification method presented in this Letter.

We do not ignore that a more general situation could involve the contribution of several competing nonconservative processes. Yet the simulations show that the resulting dynamic-dissipation curves could be considered a combination of the individual dissipative processes which in turns allows us to determine each respective contribution from the whole curve. Finally, the quantitative agreement obtained between simulations and experiments could serve as an initial step to develop a dissipation spectroscopy for quantitative analysis of material properties.

This work was financially supported by the European Commission (FORCETOOL, NMP4-CT-2004-013684).

*Corresponding author.

Electronic address: rgarcia@imm.cnm.csic.es

[1] R. W. Carpick and M. Salmeron, Chem. Rev. 97, 1163 (1997).

[2] A. N. Cleland, Foundations of Nanomechanics (SpringerVerlag, Berlin, 2003).

[3] E. Meyer, H. J. Hug, and R. Bennewitz, Scanning Probe Microscopy (Springer-Verlag, Berlin, 2004).
[4] B. Gotsmann, C. Seidel, B. Anczykowski, and H. Fuchs, Phys. Rev. B 60, 11051 (1999).

[5] M. F. Yu, T. Kowalewski, and R. S. Ruoff, Phys. Rev. Lett. 86, 87 (2001).

[6] P. M. Hoffmann, S. Jeffery, J. B. Pethica, H. Ozgür, and A. Oaral, Phys. Rev. Lett. 87, 265502 (2001).

[7] M. Stark, C. Möller, D. J. Müller, and R. Guckenberger, Biophys. J. 80, 3009 (2001).

[8] M. Balantekin and A. Atalar, Phys. Rev. B 67, 193404 (2003).

[9] P. Martin, S. Marsaudon, J. P. Aimé, and B. Bennetau, Nanotechnology 16, 901 (2005).

[10] W. A. Hofer, A. S. Foster, and A. L. Shluger, Rev. Mod. Phys. 75, 1287 (2003).

[11] F. J. Giessibl, Rev. Mod. Phys. 75, 949 (2003).

[12] R. Garcia, J. Tamayo, and A. San Paulo, Surf. Interface Anal. 27, 312 (1999).

[13] B. Anczykowski, B. Gotsman, H. Fuchs, J. P. Cleveland, and V. B. Elings, Appl. Surf. Sci. 140, 376 (1999).

[14] M. J. D’ Amato, M. S. Markus, M. A. Eriksson, and R. W. Carpick, Appl. Phys. Lett. 85, 4738 (2004).

[15] N. Rehse, S. Marr, S. Scherdel, and R. Magerle, Adv. Mater. 17, 2203 (2005).

[16] W. Wu, K. Matyjaszewski, and T. Kowalewski, Langmuir 21, 1143 (2005).

[17] H. Bodiguel, H. Montes, and C. Fretigny, Rev. Sci. Instrum. 75, 2529 (2004).

[18] P. D. Ashby and C. M. Lieber, J. Am. Chem. Soc. 127, 6814 (2005).

[19] T. R. Rodriguez and R. Garcia, Appl. Phys. Lett. 84, 449 (2004).

[20] L. N. Kantorovich and T. Trevethan, Phys. Rev. Lett. 93, 236102 (2004).

[21] N. Oyabu, P. Pou, Y. Sugimoto, P. Jelinek, M. Abe, S. Morita, R. Perez, and O. Custance, Phys. Rev. Lett. 96, 106101 (2006).

[22] A. Schirmeisen and H. Hölscher, Phys. Rev. B 72, 045431 (2005).

[23] H. Yoshizawa, Y.-L. Chen and J. Israelachvili, J. Phys. Chem. 97, 4128 (1993).

[24] S. Hu and A. Raman, Phys. Rev. Lett. 96, 036107 (2006).

[25] T. R. Rodriguez and R. Garcia, Appl. Phys. Lett. 80, 1646 (2002).

[26] B. V. Derjaguin, V. M. Muller, Y. P. Toporov, J. Colloid Interface Sci. 53, 314 (1975).

[27] J.E. Sader, J. W. M. Chon, and P. Mulvaney, Rev. Sci. Instrum. 70, 3967 (1999).

[28] J. Israellachvili, Intermolecular and Surface Forces (Academic, London, 1992).

[29] K. Tanaka et al., Macromolecules 29, 3040 (1996).

[30] N.F. Martinez and R. Garcia, Nanotechnology 17, S167 (2006). The coexistence of attractive and repulsive interaction regimes in AM-AFM is a nonlinear dynamics property. Those transitions can be explained by using tip-surface conservative interactions.

[31] The DMT model is not considered suitable for simulating mechanical properties of very compliant material such as PB $(Y \sim 1-3 \mathrm{MPa})$.

[32] M.S. Marcus, R. W. Carpick, D. Y. Sasaki, and M. A. Eriksson, Phys. Rev. Lett. 88, 226103 (2002). 